\title{
TEMAS Y DEBATES EN LA HISTORIA DE LA VIOLENCIA POLÍTICA EN CHILE
}

\section{Igor Goicovic Donoso (USACH)}

Departamento de Historia, Universidad de Santiago de Chile Av. Libertador Bernardo O’Higgins, 3363, Santiago de Chile igor.goicovic@usach.cl

\section{Resumen:}

En este artículo identificamos, analizamos y comentamos las principales contribuciones que desde el campo historiográfico han abordado el fenómeno de la violencia política insurgente en Chile, para el ciclo histórico 1973-1994. Para ello nos hemos centrado en aquellos trabajos que estudian, en particular, al Movimiento de Izquierda Revolucionaria (MIR); la política de rebelión popular de masas del Partido Comunista (PC), expresada en su brazo armado, el Frente Patriótico Manuel Rodríguez (FPMR); y al Complejo MAPU Lautaro, una organización política de origen popular y cristiano, que constituyó una clara ruptura con las formas de hacer política en Chile. En este trabajo se da cuenta de los principales campos, aspectos y tesis desarrollados por esta historiografía y se señalan aquellas áreas que requieren de mayor profundización.

\section{Palabras Clave:}

Historiografía - Violencia - Política - Dictadura - Chile

\begin{abstract}
:
In this article we identify, analyze and discuss the main contributions from the historiographical field have addressed the phenomenon of insurgent political violence in Chile, 1973-1994 for the historical cycle. For this purpose we have focused on studying those works, in particular the Revolutionary Left Movement (MIR ); policy of mass popular rebellion of Communist Party (PC), expressed in its armed wing, the Manuel Rodriguez Patriotic Front (FPMR ); Complex and MAPU Lautaro, a political organization and popular Christian origin, which was a clear break with the ways of politics in Chile. This paper realizes the main areas, aspects and developed by this thesis historiography and realizes areas that require greater depth.
\end{abstract}

\section{Keywords:}

Historiography - Violence - Policy - Dictatorship 


\title{
TEMAS Y DEBATES EN LA HISTORIA DE LA VIOLENCIA POLÍTICA EN CHILE*
}

\author{
Igor Goicovic Donoso (USACH)
}

igor.goicovic@usach.cl

\section{Presentación}

La violencia política en Chile ha sido un tema escasamente abordado por la historiografía, y el déficit es aún mayor cuando se analiza este fenómeno para la historia reciente. Ello no significa que la violencia carezca de investigaciones científicas; pero han sido la politología y en menor medida la sociología, las disciplinas que se han preocupado de este campo de estudio. Es más, los problemas asociados a la historia reciente del país han tenido en el periodismo de investigación su principal área de desarrollo.

No obstante, la violencia política aparece como un tema recurrente tanto en la discusión pública, como en la comunicación social. Así, la justificación que hacen las instituciones castrenses o la explicación que elaboran las comisiones oficiales formadas por el Estado, respecto del Golpe de militar del 11 de septiembre de 1973, se encontrarían en los hechos de violencia política que se sucedieron en Chile a partir de 1967. Mientras que las dos visiones predominantes sobre las violaciones a los derechos humanos cometidas por la dictadura militar, nos indican que éstas fueron el resultado de los excesos de algunos funcionarios del régimen o de la aplicación de una política de terrorismo de Estado.

Por su parte, las acciones de insurgencia armada del ciclo 1983-1987 se explicarían por la ejecución de la política de rebelión popular enunciada por el PC, a comienzos de la década de 1980. Mientras que la violencia política producida durante la transición a la democracia (19901994), estaría dando cuenta de la emergencia en Chile del flagelo del terrorismo.

Más recientemente, en el contexto de las movilizaciones sociales que han afectado al país desde el año 2006, el fenómeno de la violencia vuelve a emerger vinculado, esta vez, a la irrupción de grupos de inadaptados o, en el mejor de los casos, anarquistas. ${ }^{1}$

No cabe duda, entonces, que este es un tema de gran relevancia. En especial cuando se trata de analizar la historia de Chile de estas últimas cinco décadas. Y, a pesar de ello, reiteramos, es un tema escasamente abordado por la historiografía. ${ }^{2}$

\footnotetext{
Una versión preliminar de este trabajo fue presentada en Segundo Seminario Internacional sobre Historia de la Violencia en América Latina. Siglos XIX y XX, realizado en la Universidad Nacional de Córdoba, Argentina, los días 30 y 31 de octubre de 2014. El artículo forma parte del proyecto de investigación Contexto bistórico y dinámicas políticas de la insurgencia armada en Chile (1978-1994), FONDECY'T N 1130323.

${ }^{1}$ La memoria del "pasado violento" como un fenómeno que tensiona las relaciones políticas en el presente, se puede analizar en Lefranc, Sandrine, "Aquello que no se conmemora. ¿Democracias sin un pasado compartido?, Revista de Ciencia Política, 23: 2, Santiago de Chile, 2003, pp. 231-240.

2 Un primer balance historiográfico sobre estos aspectos lo aporta Herrera, Patricio, "La vía revolucionaria en Chile. Entre democracia, dictadura y transición (1965-1994)”, en Oikión, Verónica, Rey, Eduardo y López, Martín (Editores), El estado de las luchas revolucionarias en América Latina (1959-1996). Estado de la cuestión, Santiago de Compostela: El Colegio de Michoacán - Universidad de Santiago de Compostela, 2014, pp. 165-184.
} 
En este artículo nos proponemos analizar la producción historiográfica referida la violencia política protagonizada en Chile por las organizaciones de la denominada "izquierda revolucionaria", en el ciclo histórico 1978-1994. ${ }^{3}$

Durante este período es posible observar un creciente desarrollo de la violencia política, tanto en la frecuencia alcanzada por las acciones de esta naturaleza, como en los altos niveles de especialización operados por los grupos armados al objeto de desplegar dichas acciones. Ello se corresponde con un enunciado discursivo que legitimaba el uso de la violencia política contra la dictadura militar, como con un diseño estratégico y táctico que viabilizaba el uso de la misma como recurso de la acción política. A ello habría que sumar (en su etapa más álgida), un escenario de efervescencia social (el ciclo de protestas populares de 1983 a 1987), que estimuló el despliegue de formas primarias (o milicianas) de violencia política. ${ }^{4}$

A nuestro juicio, el estudio histórico de estos fenómenos y de su incidencia en la conformación del escenario político del país en la década de 1980, así como sus proyecciones hacia la primera fase de la transición a la democracia, es de gran relevancia para el conocimiento del pasado reciente de Chile y para la comprensión de las tensiones y conflictos que enfrenta la sociedad chilena contemporánea.

\section{Perspectivas generales sobre la violencia política en Chile}

Una de las imágenes más extendidas respecto de América Latina, es su condición de región estructuralmente sacudida por diferentes manifestaciones de violencia. Desde la violencia criminal protagonizada por el narcotráfico (que tiene en la desaparición de 43 jóvenes en el Estado de Guerrero en México, su manifestación más brutal y contingente), hasta las violencias domésticas (como el femicidio) o las interpersonales (como las riñas con resultado de muerte). ${ }^{5}$

En este contexto el estudio de la violencia política ha considerado tanto la violencia represiva, que adquirió particular notoriedad en la década de 1970, como las manifestaciones más radicales de la insurgencia armada. ${ }^{6}$

\footnotetext{
${ }^{3}$ Utilizamos restrictivamente el concepto de "izquierda revolucionaria" para referir a las organizaciones políticas del período 1978-1990, que pretendieron derrocar la dictadura militar mediante el uso de la violencia política: Partido Comunista (PC), Movimiento de Izquierda Revolucionaria (MIR) y MAPU-Lautaro. El Partido Socialista (PS), bajo el liderazgo de Clodomiro Almeyda Medina, puede ser situado también en esta clave; en especial si tenemos en cuenta que su estrategia política, a partir de 1979, se definía "con perspectiva insurreccional". No obstante este referente no desarrolló, prácticamente, operaciones armadas en el ciclo en estudio, de manera que lo hemos excluido de este análisis. Al respecto ver, Gutiérrez, Eduardo, Ciudades en las sombras (Una bistoria no oficial del Partido Socialista de Chile, 1970-1990), Santiago de Chile: PUBLIPROM, 2003 y Ortiz, Edison, El socialismo chileno: De Allende a Bachelet (1973-2005), Santiago de Chile: FADELSO, Prensa Latinoamericana e Instituto Chileno de Estudios Humanísticos, 2007.

${ }^{4}$ La relación entre la violencia política y las "ideas, ideales e idealizaciones" que la justifican, se encuentra tratada en Balibar, Étienne, Violencias, identidades y civilidad. Para una cultura politica global, Barcelona: Gedisa Editorial, 2005, pp. 101-120.

${ }^{5}$ Ver, al respecto, el reciente trabajo de compilación de Ansaldi, Waldo y Giordano, Verónica, América Latina: Tiempos de violencia, Buenos Aires: Ariel, 2014

${ }^{6} \mathrm{El}$ tema de la violencia represiva ha sido ampliamente tratado por la literatura especializada. El contexto más amplio de la definición y aplicación de la Doctrina de Seguridad Nacional se encuentra en Velásquez, Edgard, "Historia der la doctrina de la seguridad nacional", Convergencia. Revista de Ciencias Sociales, 9: 27, México D.F., 2002, pp. 11-39. Un estudio construido sobre la base de los informes de las comisiones Rettig y Valech en Hiner, Hillary, "Voces soterradas, violencias ignoradas: Discurso, violencia política y género en los informes Rettig y Valech", Latin American Research Review, 44: 3, Pittsburgh, 2009, pp. 50-74. En relación con la insurgencia armada una mirada de conjunto, desde su ascenso a partir de 1959 hasta su actual declinación, en la compilación de Pozzi, Pablo y Pérez, Claudio (Editores), Por el camino del Che. Las guerrillas latinoamericanas, 1959-1990, Buenos Aires: UBA - UAHC RELAHO - Imago Mundi, 2011. Una problemática similar, pero bajo el enfoque de terrorismo, en Feldmann, Andreas, Feldmann, Andreas, "A shift in the paradigm of violence. Non-governmental terrorism in Latin America
} 
No obstante, en Chile, el problema de la violencia política ha sido abordado sólo de manera periférica en los estudios sobre la Unidad Popular (UP), la dictadura militar y la transición a la democracia. ${ }^{7}$ Este es el caso de las historias generales de Sofía Correa et al y de Gabriel Salazar y Julio Pinto. ${ }^{8}$ Esto resulta sorprendente ya que en la región los estudios de esta naturaleza son abundantes y sistemáticos.

Una parte de los estudios elaborados en el país, especialmente en el campo de la politología y de la sociología, como el de Martínez, Tironi y Weinstein y el de José Weinstein, han caracterizado la violencia como "reventones" espontáneos, devenidos de conductas anómicas. Por lo tanto, sin mayor incidencia en la construcción de la sociedad contemporánea. ${ }^{9}$ En este enfoque, las situaciones de discriminación estructural que afectaban a los jóvenes en la década de 1980: Económica, social, política, cultural y generacional, se sumaban a un contexto económico complejo (crisis económica de 1982), que dificultaba los procesos de integración de estos sujetos. Estos factores habrían operado como catalizadores de la participación de los jóvenes en las protestas populares del ciclo 1983-1987, pero sólo como expresión de rabia y frustración. ${ }^{10}$ Los vínculos a nivel territorial de este segmento juvenil con las organizaciones de la izquierda revolucionaria no son identificados. Los autores desconocen que a partir de las comunidades cristianas de base, fuertemente desarrolladas en las poblaciones desde comienzos de la década de 1960, se inició el proceso de reconstrucción tanto del tejido social popular, como de las organizaciones de la izquierda. Este proceso inaugurado hacia 1975, ya se encontraba ampliamente desarrollado para 1983. La articulación de la juventud popular en torno a las comunidades cristianas de base y a las organizaciones políticas de la izquierda (en especial el PC y el MIR), le otorgó centralidad estratégica y conducción política a la protesta radical. ${ }^{11}$

since the end of the Cold War", Revista de Ciencia Politica, 25: 2, Santiago de Chile, 2005, pp. 3-36. El balance historiográfico más actualizado sobre estos temas en Oikión, Verónica, Rey, Eduardo y López, Martín (Editores), El estado de las luchas revolucionarias en América Latina (1959-1996). Estado de la cuestión, Santiago de Compostela: El Colegio de Michoacán - Universidad de Santiago de Compostela, 2014.

${ }^{7}$ La dictadura militar chilena (1973-1990) y la transición temprana a la democracia (1990-1994), como campos de estudio, cuentan con una amplia literatura. En nuestros trabajos sobre la materia referimos parte importante de esas contribuciones. Ver, al respecto, Goicovic Donoso, Igor "Transición y violencia política en Chile (1988-1994)", Ayer. Revista de Historia Contemporánea, 79: 3, Madrid, 2010, pp. 59-86; Goicovic Donoso, Igor (2010b), "La transición política en Chile. Especificidades nacionales y puntos de referencia con el caso español (1988-1994)", en Martín, Oscar y Ortiz, Manuel (Coordinadores), Claves internacionales en la transición española, Madrid: Los Libros de la Catarata, 2010, 288-319; y Goicovic Donoso, Igor, "La implacable persistencia de la memoria. Reflexiones en torno al Informe de la Comisión de Prisión Política y Tortura", Revista de Historia Actual, 2: 2, Cádiz, 2004, pp. 73-91.

${ }^{8}$ Correa, Sofía et al, Historia del siglo XX chileno: Balance paradojal, Santiago de Chile: Editorial Sudamericana, 2011 y Salazar, Gabriel y Pinto, Julio, Historia contemporánea de Chile. Estado, legitimidad y ciudadanía, vol. 1, Santiago de Chile: LOM Ediciones, 1999.

${ }_{9}^{9}$ Martínez, Javier, Tironi, Eugenio y Weinstein, Eugenia, La violencia en Chile. Personas y escenarios de la violencia colectiva, Santiago de Chile: Ediciones Sur, 1990 y Weinstein, José, Los jóvenes pobladores en las protestas nacionales (1983-1984). Una visión socio-politica, Santiago de Chile: CIDE, 1989.

${ }^{10}$ En este punto los autores siguen el enfoque de Ted Robert Gurr sobre la "carencia relativa", que instala un punto de inflexión entre las expectativas de mejoramiento de las condiciones de vida de los sujetos y sus posibilidades reales de acceder a los bienes y servicios a los cuales creen tener derecho. Ver, sobre este tema, Gurr, Ted Robert, Why men rebel? Princeton: Princeton University Press, 1970 y Gurr, Ted Robert, "Why minorities rebel: A global analysis of communal mobilization and conflict since 1945”, International Political Science Review, 14: 2, Canberra - Nueva York, 1993, pp. 161-201.

11 El tema de las comunidades cristianas de base y su relación con la reconstrucción del tejido social y político popular, no ha concitado el interés de los investigadores. Las referencias sobre la materia tratan, de manera general, la relación entre la Iglesia y el campo popular (ver, por ejemplo, Salinas Campos, Maximiliano, Historia del pueblo de Dios en Chile: La evolución del cristianismo desde la perspectiva de los pobres, Santiago de Chile: Rehue, 1987 y CEDM, Comunidades eclesiales de base, 20 años en Chile: Evaluación y perspectivas, Santiago de Chile: Centro Ecuménico Diego de Medellín, 1989); o abordan las orientaciones doctrinarias que la Iglesia le asignó a esta práctica (ver, Barrios Valdés, Marciano, La espiritualidad católica chilena en tiempos del Vaticano II, Santiago de Chile: Universidad Católica Blas Cañas, 1996). La dimensión política del fenómeno sólo se encuentra en el trabajo de Ortiz Barrios, Johanna, Las comunidades cristianas de 
Otro grupo de trabajos proviene de los centros de investigación vinculados al pensamiento conservador, ello a objeto de vincular la violencia política con la noción de terrorismo. ${ }^{12}$ En el caso de la historiadora Patricia Arancibia Clavel, el esfuerzo se orienta a demostrar que la violencia política en Chile arrancó a mediados de la década de 1960, que fue el resultado de la influencia que alcanzó la Revolución Cubana al interior de la izquierda chilena, que operó primero como radicalización del discurso y, posteriormente, como radicalización de la acción política. Por esta vía, concluye la autora, la violencia política no parte con la dictadura militar, sino que con los discursos y acciones violentistas de la izquierda, que desembocaron en una espiral de radicalización política, que llevó luego al "pronunciamiento militar". ${ }^{13}$

Cabe consignar que en Chile, a diferencia de lo ocurrido en otros países de la región, como Argentina, tras la caída del gobierno de Juan Domingo Perón (1955) o Colombia, después del asesinato de Jorge Eliecer Gaitán (1948), la violencia política aguda tendió a convertirse en un fenómeno recurrente sólo después del golpe de Estado de 1973. Es evidente que en el ciclo previo, 1967 a 1973, los enfrentamientos sociales derivaron hacia una creciente polarización en la cual los enfrentamientos violentos operaron como rasgo distintivo, pero el uso de la violencia de manera sistemática para aplastar al adversario (dictadura) o para combatir al régimen de facto (insurgencia), recién se desplegó tras la intervención militar de $1973 .{ }^{14}$ Aún más, es posible indicar que el ciclo se inició hacia 1978, pero adquirió mayor envergadura y extensión con el desarrollo de las protestas populares entre 1983 y $1987 . .^{15}$

En ese sentido, el desarrollo experimentado en Chile por la "nueva historia política", sobre la base de la recuperación de la historia del tiempo presente, de las redes y relaciones de poder, así como del militantismo y la construcción sociocultural de lo político, ha permitido desarrollar

base como manifestación de la iglesia popular chilena, Tesis de Periodismo, Santiago de Chile: Universidad de Santiago de Chile, 2000.

${ }^{12}$ Este enfoque se encuentra ampliamente desarrollado en Burleigh, Michael, Sangre y rabia. Una historia cultural del terrorismo, Madrid: Editorial Taurus, 2008. Los enfoques contemporáneos, afectos al "síndrome del 11/9", se encuentran correctamente expuestos en, Kurrild-Klitgaard, Peter, Justesen, Mogens K y Klemmensen, Robert, "The political economy of freedom, democracy and transnational terrorism", Public Choice, 128, New York, 2006, pp. 289315. La reinterpretación "local” de este enfoque en Godoy, Oscar, "Terrorismo e historia”, Estudios Públicos, 84, Santiago de Chile, 2001, pp. 33-47. El primer analista en rotular como acciones terroristas las acciones contra la dictadura desplegadas por el MIR, fue Andrés Benavente. Ver, Benavente Urbina, Andrés, "Movimiento de Izquierda Revolucionaria: Trayectoria y presente”, Revista Política, 12, Santiago de Chile, 1987, pp. 121-155.

13 Ver, Arancibia, Patricia et al, Los hechos de violencia en Chile. Del discurso a la acción, Santiago de Chile: Universidad Finis Terrae - Libertad y Desarrollo, 2003; Arancibia, Patricia, Los orígenes de la violencia política en Chile, 1960-1973, Santiago de Chile: Universidad Finis Terrae - Libertad y Desarrollo, 2001.

${ }^{14}$ Las excepciones la constituyen el MIR, que entre 1969 y 1970 asaltó varias instituciones financieras; el Frente Nacionalista Patria y Libertad (FNPL), organización de extrema derecha involucrada en sabotajes, violencia urbana y en el asesinato del edecán naval del Presidente Salvador Allende, capitán Arturo Araya Peters (1973); y la Vanguardia Organizada del Pueblo (VOP). Como veremos más adelante, la bibliografía referida al MIR es bastante amplia. Pero sobre el FNPL no hay un trabajo específico. La mejor referencia se encuentra en Valdivia, Verónica, Nacionales y gremialistas. El parto de la nueva derecha política chilena, 1964-1973, Santiago de Chile: LOM Ediciones, 2008. Mientras que la VOP cuenta con sólo un estudio preliminar, Ver, Pomar Rodríguez, Jorge, "La Vanguardia Organizada del Pueblo (VOP): Origen, subversión y aniquilamiento. ¡El pan que con sangre fue quitado, con sangre será recuperado!”, XIV Encuentro de Latinoamericanistas Españoles, Universidad de Santiago de Compostela: Santiago de Compostela, 2010, pp. 1496-1506.

15 El tema de las protestas populares tampoco cuenta con estudios monográficos relevantes. Se puede conocer el fenómeno a través de los textos de Salazar, Gabriel, Violencia política popular en las 'grandes alamedas' (1947-1987), Santiago de Chile: Ediciones SUR, 1990 y de De la Maza Gonzalo y Garcés, Mario, La explosión de las mayorías. Protesta nacional, 1983-1984, Santiago de Chile: ECO, 1985. 
nuevas e interesantes contribuciones al tema. ${ }^{16}$ No obstante, la investigación referida a las organizaciones político-militares, que son objeto de este estudio, muestra diferencias notables. ${ }^{17}$

\section{Los estudios sobre el MIR}

El MIR ha sido, sin duda, el referente que ha concitado mayor atención e interés entre los investigadores. Su irrupción temprana, en una sociedad que se reivindicaba a sí misma como democrática y estable, así como su activa presencia política en el ciclo 1970-1973, explican en gran medida esta especial preocupación. Cabe destacar que los trabajos más sistemáticos y rigurosos sobre el MIR cubren, principalmente, su período de fundación y organización temprana (1965-1969) y su desarrollo político durante la Unidad Popular (1970-1973).

Respecto de la primera etapa en la historia del MIR (1965-1969), el trabajo más reciente es el de la historiadora griega, Eugenia Palieraki. ${ }^{18}$ Un trabajo que surge de la revisión de un amplio volumen de fuentes documentales, impresas y orales y que, en consecuencia, se convierte en el resultado más acabado respecto del MIR en este período. No obstante, este texto abre una serie de controversias que a mi juicio no quedan lo suficientemente esclarecidas. En relación con los antecedentes del MIR, la autora establece que esta organización fue el resultado de una cultura política "de la extrema izquierda", articulada desde comienzos de la década de 1920 y asociada, ineludiblemente, a la trayectoria política de sus fundadores. Ellos constituirían La vieja Guardia Mirista. Es por ello que los fundamentos políticos en los cuales asentaron sus prácticas estos sujetos y sus respectivas trayectorias, explicarían su concurrencia al hito fundacional. Por lo anterior, el contexto histórico en el cual se fundó el MIR (es decir la contingencia de la década de 1960), carecería de relevancia.

Para afirmar su postura frente al tema la autora realiza una reconstrucción biográfica de varios dirigentes del primer MIR, entre los cuales destaca la figura de Clotario Blest y junto con él la influencia del cristianismo revolucionario. Pero en este caso en particular, el énfasis interpretativo se encuentra en el anti partidismo de Blest, con lo cual queda subsumida la postura clasista del dirigente, la cual también influyó fuertemente en su concurrencia a la formación de la nueva organización.

Más adelante, Eugenia Palieraki, estudia la influencia del trotskismo en la fundación y primeros pasos del MIR, destacando la participación de Oscar Waiss, Enrique Sepúlveda, Humberto Valenzuela y Luis Vitale. No cabe duda del aporte que los trotskistas hicieron al MIR, en especial a nivel intelectual y en sus primeros vínculos con organizaciones de masas; este aporte resultó fundamental para su desarrollo político ulterior, especialmente en los denominados frentes

16 Ver, al respecto, Sirinelli, Jean-François, "El retorno de lo político", Revista de Historia Contemporánea, 9, Bilbao, 1993, pp. 25-35.

${ }^{17}$ En este artículo aludimos de manera exclusiva a los trabajos científicos. Existe una amplia gama de memorias y testimonios que se han editado en estas últimas dos décadas que, sin lugar a dudas, constituyen una fuente valiosa de información. Pero que, en cuanto tales, no pueden ser evaluados como producción científica.

18 Palieraki, Eugenia, La revolución ya viene. El MIR cbileno en los años sesenta, Santiago de Chile: LOM, 2014. Sobre esta etapa en la historia del MIR, pero con enfoques y ejercicios interpretativos diferentes ver, Salinas, Sergio, El tres letras. La historia del MIR, Santiago de Chile: RIL Editores, 2013; Vitale, Luis, Contribución a la historia del MIR (1965-1970), Santiago de Chile: Ediciones Instituto de Investigación de Movimientos Sociales Pedro Vuskovic, 1999; y Sandoval Ambiado, Carlos, MIR. Una historia, Santiago de Chile: Sociedad Editorial Trabajadores, 1990. Este período también se encuentra tratado en las siguientes tesis de grado: Valdés Navarro, Pedro, Elementos teóricos en la formación y desarrollo del MIR durante el período 1965-1970, Tesis de Licenciatura, Valparaíso: Instituto de Historia y Ciencias Sociales, Universidad de Valparaíso, 2006 y Moulian Jara, Iñaki, Contextualización, origen y evolución del Movimiento de Izquierda Revolucionaria (MIR), en Chile, 1959-1970, Tesis de Licenciatura, Valdivia: Escuela de Educación Media Científico Humanista, Universidad Austral de Chile, 2001. Un enfoque crítico sobre la política del MIR, pero con perspectiva diacrónica, se encuentra en los ensayos publicados por Hernán Vidal. Ver, Vidal, Hernán, Presencia del Movimiento de Izquierda Revolucionaria (MIR) (14 claves existenciales), Santiago de Chile: Mosquito, 1999 y El Movimiento de Izquierda Revolucionaria (MIR) de Chile en la justicia transicional, Santiago de Chile: Alternativas, 2014. 
intermedios, a nivel obrero, poblacional y campesino. Es más, muchas de las definiciones adoptadas por el MIR en su política de masas poseen una evidente influencia de esta corriente de pensamiento. De la misma manera la teoría inicial sobre la acción revolucionaria, recogida de Lenin, estaba debidamente procesada o tamizada por el enfoque trotskista. Pero no es menos efectivo que dicha influencia se vio gradualmente desplazada por el enfoque castro-guevarista, tanto por la desvinculación gradual de muchos cuadros trotskistas entre 1965 y 1968 y por las expulsiones del año 1969.

También se analiza en este libro la influencia de la disidencia comunista, en especial del Reinosismo en la formación del núcleo de fundación del MIR. En este punto la autora hace referencia a la temprana opción hecha por el dirigente comunista Luis Reinoso, por la lucha armada y los debates y controversias que este fenómeno provocó al interior del PC. Debates que concluyeron con la expulsión de Reinoso y la deriva de muchos de sus militantes a la Vanguardia Revolucionaria Marxista... donde encontraran, como dice la autora: “... jóvenes militantes, ávidos de aventuras armadas" (p. 53). Un comentario complejo que afirma viejos estereotipos instalados por el pensamiento conservador. De esta forma, para Eugenia Palieraki, la crítica a la izquierda tradicional, el rechazo al escenario electoral y la opción por la vía armada, no serían aportes novedosos del MIR. Por el contrario, serían parte del acervo político de la "extrema izquierda" que concurre a la formación de la organización. No obstante, en el tratamiento de este punto, la autora sigue la tendencia tradicional de la historiografía en cuanto a realizar un análisis de discurso, sin hacerse cargo de la trayectoria del nuevo partido. Ello le habría permitido constatar que el MIR, en cuanto "partido nuevo", surge, efectivamente, en 1967 y se desarrolla y alcanza visibilidad nacional e internacional, a partir de 1969. La novedad del MIR, entonces, no hay que buscarla en 1965, sino que a partir de 1967; y no hay que observarla exclusivamente en su dimensión discursiva, sino que más bien en su práctica política.

El desarrollo del MIR a partir de 1969 (expulsión de la disidencia trotskista) y durante el período de la Unidad Popular (1970-1973), reúne la mayor y más prolífica serie de contribuciones. ${ }^{19}$ No obstante, una parte importante de los textos editados recientemente corresponden a memorias, testimonios y balances políticos realizados por ex militantes del MIR. ${ }^{20}$

En este acápite nos vamos a referir a cinco trabajos que consideramos fundamentales. El primero de ellos es un ensayo publicado en 2003, por el ex secretario general del MIR, el sociólogo Andrés Pascal Allende. ${ }^{21}$ En este texto Andrés Pascal da cuenta de los ejes principales de la estrategia mirista en lo que esta organización denominó "la fase prerrevolucionaria". A saber, la formación de un partido de cuadros de rápido y exponencial desarrollo; la acumulación de fuerzas social, política e ideológica a través de los denominados "frentes intermedios"; 22 y la

\footnotetext{
19 Entre las compilaciones más importantes es necesario destacar las realizadas por, Sandoval Ambiado, Carlos, Movimiento de Irquierda Revolucionaria. 1970-1973. Coyunturas, documentos y vivencias, Concepción: Escaparate, 2004; Hernández Vásquez, Martín, El pensamiento revolucionario de Bautista van Schouwen, 1943-1973, Concepción: Escaparate, 2004; Naranjo, Pedro et al (Compiladores), Miguel Enríquez: El proyecto revolucionario en Chile, Santiago de Chile: LOM, 2004; Radrigán, Cecilia y Ortega, Myriam (Compiladoras), Miguel Enríquez: Con vista a la esperanza, Concepción: Escaparate, 1998; y MIR, V arios. Movimiento de Izquierda Revolucionaria chilena, Madrid: Editora Zero, 1976. ${ }^{20}$ Ver al respecto, Bravo Aguilera, José Manuel, De Carranco a Carrán. Las tomas que cambiaron la historia, Santiago de Chile: LOM, 2012; García Concha, Enérico, Todos los días de la vida. Recuerdos de un militante del MIR chileno, Santiago de Chile: Cuarto Propio, 2010; Marambio, Max, Las armas de ayer, La Habana: Editorial José Martí, 2008; Rodríguez, Guillermo, De la brigada secundaria al cordón Cerrillos, Santiago de Chile: Universidad Bolivariana, 2007; y Pérez, Enrique, La búsqueda interminable. Diario de un exiliado político chileno en Suecia, Santiago de Chile: Mosquito Comunicaciones, 1996.

${ }^{21}$ Pascal Allende, Andrés, El MIR chileno. Una experiencia revolucionaria, Buenos Aires: Editorial Cucaña, 2003.

22 Hacia 1970 el trabajo de masas del MIR se operacionalizó a través de los denominados "frentes intermedios", estructuras parapartidarias, formadas por simpatizantes y ayudistas del MIR, que permitían una relación más estrecha con las organizaciones de base. Entre estos frentes intermedios se encontraban, el Frente de Trabajadores Revolucionarios (FTR), el Movimiento Campesino Revolucionario (MCR), el Movimiento de Pobladores
} 
preparación del movimientos de masas y de la organización partidaria para el inevitable enfrentamiento armado con la burguesía. En este último punto la estrategia de guerra popular suponía, necesariamente, la "fractura por abajo" de las fuerzas armadas y la movilización hacia el campo popular y revolucionario, de los conscriptos y clases de las fuerzas armadas. Concluye el ensayo señalando que la ofensiva contrarrevolucionaria desatada a partir de marzo de 1973 no logró ser contenida y que la política reformista del Gobierno de la Unidad Popular (UP) y las debilidades del campo revolucionario permitieron el éxito de la asonada golpista.

Para Julio Pinto Vallejos, la ruptura al interior del campo popular entre "gradualistas" y "rupturistas", en función de los contenidos y formas de la "revolución", explica el fracaso de ambos y el triunfo de la reacción burguesa. ${ }^{23}$ En este diagnóstico Pinto Vallejos reconoce tres ejes controversiales entre "gradualistas" y "revolucionarios": Las vías para llegar al socialismo, entendidas como los ritmos y los tiempos del proceso; el carácter local o latinoamericano del mismo; y por último la construcción social del poder revolucionario, que los "gradualistas" reconocían al interior de la institucionalidad política y los "rupturistas" pretendían construirlo al interior de las masas, en el denominado "poder popular". No obstante, señala Pinto, la derrota de 1973, golpeó a ambas tendencias por igual, supuso el reflujo del movimiento de masas y liquidó la utopía revolucionaria.,

Por su parte, Sebastián Leiva, al estudiar la construcción del poder popular en Chile y Argentina, entre 1970 y 1976, enfatiza que, en el caso chileno, el desarrollo de la teoría del "poder dual" de Lenin, es el resultado de las disquisiciones teóricas realizadas por la Comisión Política y el Comité Central del MIR y en particular por los aportes al tema de Miguel Enríquez, pero en estrecha articulación con las experiencias de masas desplegadas por el partido en la base del movimiento popular. ${ }^{24}$ Desde esta perspectiva, para Leiva, lo que opera es una síntesis dialéctica entre conducción política y autonomía social. ${ }^{25}$

Una de las experiencias más exitosas de construcción de poder popular liderada por el MIR durante este período corresponde al campamento Nueva La Habana situado en la zona oriente de

Revolucionarios (MPR), el Movimiento Universitario de Izquierda (MUI), y el Frente de Estudiantes Revolucionarios (FER).

${ }^{23}$ Pinto Vallejos, Julio, "Hacer la revolución en Chile", en Pinto Vallejos, Julio (Editor), Cuando bicimos historia. La experiencia de la Unidad Popular, Santiago de Chile: LOM, 2005, pp. 9-33. La tendencia "gradualista", representada genéricamente por la UP, tenía como referentes fundamentales al PC, un sector del PS, el Partido Radical (PR) y el MAPU Obrero y Campesino; todos ellos bajo el liderazgo de Salvador Allende. Mientras que la tendencia "rupturista" la encabezaba el MIR, y formaban parte de la misma el sector mayoritario del PS, el MAPU dirigido por Oscar Guillermo Garretón y la Izquierda Cristiana. La reflexión teórica sobre este tema, desde la intelectualidad mirista en, Marini, Ruy Mauro, El reformismo y la contrarrevolución. Estudios sobre Chile, México D.F.: ERA, 1976.

${ }^{24}$ La contribución de Miguel Enríquez a la construcción teórica, política y operativa del MIR, en Amorós, Mario, Miguel Enríquez. Un nombre en las estrellas. Biografía de un revolucionario, Santiago de Chile: Ediciones B, 2014. El rol de los intelectuales latinoamericanos en el debate teórico y político al interior del MIR lo ha trabajado recientemente Ivette Lozoya. Ver, al respecto, Lozoya López, Ivette, "Pensar la revolución: Pensamiento latinoamericano e intelectuales en el MIR chileno, 1965-1973. Propuesta teórica y metodológica para su estudio desde la historia intelectual y la historia de la violencia", Revista de Humanidades, 27, Santiago de Chile, 2013, pp. 173-197. Un enfoque peculiar a la relación entre elaboración de teoría, dirección política y militancia partidaria, lo aporta Julián Bastías. En su trabajo este autor sostiene que la adhesión a la "microideología" y a la conducción de los líderes inhibió en el MIR el libre desarrollo de la crítica entre la militancia. Ver, Bastías Rebolledo, Julián, “A propósito del MIR chileno. Un intento de psicología partidaria. Representaciones sociales y subsistemas ideológicos como factores de inhibición en la crítica de los militantes", en DÁdamo, Orlando; García, Virginia; y Montero, Maritza (compiladores), Psicología de la acción política, Buenos Aires: Paidos, 1995, pp. 162-203.

${ }^{25}$ Leiva Flores, Sebastián, Revolución socialista y poder popular. Los casos del MIR y del PRT-ERP, 1970-1976, Concepción: Escaparate, 2010, pp. 49-124. Las tesis sobre el poder popular en Chile y el rol del MIR en su desarrollo, durante el ciclo 1970-1973, también se encuentran desarrolladas en, Gaudichaud, Franck, Poder popular y cordones industriales: Testimonios sobre el movimiento popular urbano, 1970-1973, Santiago de Chile: LOM, 2004; y Cancino Troncoso, Hugo, Chile: La problemática del poder popular en el proceso de la Via Chilena al Socialismo, 1970-1973, Copenhague: Aarhus University Press, 1988. Las relaciones internacionales del MIR, han sido tratadas por Osvaldo Torres. Ver, Torres, Osvaldo, Democracia y lucha armada. MIR y MLN Tupamaros, Santiago de Chile: Pehuén, 2012. 
Santiago, en la actual comuna de La Florida. Al respecto, señala Boris Cofré Schmeisser, en este espacio las tesis del MIR sobre la formación del "poder dual" ("poder popular teórico"), se vieron confrontadas con la práctica concreta desplegada por los pobladores ("poder popular histórico"). ${ }^{26}$ En el ejercicio interpretativo propuesto por Cofré, ambas concepciones aparecen disociadas, no obstante la condición de militante sociales y a la vez de militantes políticos de los sujetos permite presumir que el vínculo entre la organización (el MIR) y el movimiento de pobladores en Nueva La Habana, era bastante más consistente que aquel que le supone el autor del texto y su prologuista (el historiador Mario Garcés). ${ }^{27}$

El cierre de la experiencia de la Unidad Popular, la trama conspirativa que rodeo el golpe de Estado del 11 de septiembre de 1973 y las insuficiencias de la política de resistencia desplegada por los revolucionarios frente a la intervención de los militares, se encuentra ampliamente descrita y analizada en un reciente libro de Ignacio Vidaurrázaga. ${ }^{28}$ En este texto Vidaurrázaga indica que el MIR logró reunir a su comisión política a tempranas horas de la mañana a efectos de desarrollar acciones de resistencia frente al golpe. No obstante, un componente fundamental del diseño, el apoyo de contingentes de las fuerzas armadas no se alcanzó. Producto de ello la resistencia sólo se expresó de manera esporádica y focalizada. Como no se logró revertir el golpe de Estado, la dirección del MIR acordó replegar a los cuadros del partido a la clandestinidad.

El período que se inauguró con el golpe de Estado de 1973 y que concluyó con la fragmentación y extinción del MIR (1994), cuenta con menos trabajos disciplinarios. En un texto de 2004 Cristian Pérez analizó el ciclo de persecución y aniquilamiento del MIR (1973-1975). ${ }^{29}$ En su estudio el autor evalúa críticamente la política refractaria del MIR frente al asilo, señalando que al no poseer la organización eficientes condiciones de clandestinidad, esta política terminó contribuyendo a la efectividad del accionar represivo. Sobre esa base, sostiene, la Dirección de Inteligencia Nacional (DINA), fue capturando militantes de la organización y, mediante el ejercicio de la tortura, accediendo a información sobre los cuadros de dirección del MIR. Ello, a su vez, explicaría las sucesivas caídas que afectaron tanto al Comité Central como a la Comisión Política del MIR, entre diciembre de 1973 y octubre de 1975 (Bautista Van Schouwen Arturo Villabela, Roberto Moreno, Sergio Pérez, Lumi Videla, entre otros). El punto culminante de esta escalada represiva fue la localización del Secretario General del MIR, Miguel Enríquez Espinoza, que murió en combate el 5 de octubre de 1974. Posteriormente (octubre de 1975), la dirección de relevo del MIR fue localizada en una parcela de las afueras de Santiago (Malloco), produciéndose un enfrentamiento armado en el cual murió el miembro de la Comisión Política, Dagoberto Pérez Vargas, y fueron obligados a exiliarse el dirigente Nelson Gutiérrez y el nuevo Secretario General, Andrés Pascal. Tras estos golpes represivos, concluye Cristián Pérez, el MIR prácticamente desapareció, mientras que las experiencias posteriores (1978 en adelante), asociadas a la "Operación Retorno", carecerían de relevancia política. El MIR, a su juicio, tras la muerte de Miguel Enríquez, dejó de ser lo que fue. Una conclusión sin duda interesante, ya que arranca de la constatación de una situación efectiva: El MIR perdió capacidad de intervención política en el

\footnotetext{
${ }^{26}$ Cofré Schmeisser, Boris, Campamento Nueva La Habana. El MIR y el movimiento de pobladores, 1970-1973, Concepción: Escaparate, 2007, pp. 15-32.

27 El afán de un sector de la historiografía social por construir una imagen idealizada del sujeto urbano-popular (autónomo, politizado y proyectual), no contribuye a reconocer la concurrencia de lo político militante en la construcción de las experiencias históricas del período. Se ha pretendido, de forma maniquea, introducir una hendidura entre el campo de la militancia social y el de la militancia política, que no responde a los registros que aportan las fuentes, sino que más bien, a reconocer una aparente continuidad histórica a las esencias identitarias relevadas por esta historiografía. Este enfoque ha sido sostenido por Gabriel Salazar, dando origen a una vertiente de la historia social denominada "movimientista". Ver, al respecto, Salazar Vergara, Gabriel, Movimientos sociales en Chile. Trayectoria histórica y proyección política, Santiago de Chile: Uqbar, 2012.

${ }^{28}$ Vidaurrázaga Manríquez, Ignacio, Martes once: la primera resistencia, Santiago de Chile: LOM, 2013.

${ }^{29}$ Pérez, Cristián, “Años de disparos y torturas (1973-1975). Los últimos días de Miguel Enríquez”, Estudios Públicos, 96, Santiago de Chile, 2004, pp. 355-382.
} 
ciclo 1976-1978. Pero Pérez se equivoca al suponer que el MIR desapareció, ya que se encuentra bastante acreditado que en el interior del país se constituyó un núcleo de dirección encabezado por Hernán Aguiló Martínez, que coordinaba a aproximadamente a 50 militantes, especialmente en Santiago. Mientras que varias estructuras locales y regionales, como Valdivia, Concepción y Valparaíso, si bien habían perdido vínculos con la dirección interna, mantenían un trabajo de reagrupamiento partidario en sus respectivas zonas. Por otro lado, la salida de buena parte de la dirección del partido al exterior y la excarcelación de militantes (que se replegaron a la clandestinidad o partieron al exilio), permitió el reagrupamiento de un sector importante de la militancia, que manifestó su disposición a retornar al frente interno y a las tareas partidarias. De esta manera se fueron generando, en el bienio crítico (1976-1977), las condiciones orgánicas que posteriormente permitieron el despliegue de la Operación Retorno, y la formación de un "nuevo" MIR, a partir del reclutamiento de cuadros juveniles, fogueados en la resistencia popular. ${ }^{30}$

En una perspectiva diferente, Julio Pinto Vallejos analiza el paso a la clandestinidad del MIR, poniendo énfasis en la caracterización que la organización realizó de la dictadura militar y de los posteriores procesos de reestructuración de la economía y de rediseño institucional que ésta llevó a cabo. ${ }^{31}$ En su estudio, Julio Pinto da cuenta de los intentos del MIR por mantener en funcionamiento su estructura orgánica y por recomponer sus vínculos con el movimiento de masas, de cara a la construcción de la resistencia popular. No obstante, enfatiza Pinto, la represión política del ciclo 1973-1975 obturó ambos procesos.

En este contexto, la Operación Retorno operó como un diseño estratégico que aspiraba a generar nuevas condiciones para la acumulación de fuerza social, política y militar, de cara al derrocamiento de la dictadura y la construcción de una democracia popular. No obstante las debilidades propias de la construcción de la clandestinidad del partido, así como la sobrevaloración de los niveles de reanimación del movimiento de masas, expusieron al mirismo, hacia 1982, a una nueva derrota frente a la represión.

Uno de los trabajos más completos y rigurosos sobre el MIR, en el ciclo 1978-1982, es el de Robinson Silva Hidalgo. ${ }^{32}$ En este estudio se analiza la situación del MIR después del golpe de Estado del 11 de septiembre de 1973, a efectos de explicar tanto la caracterización que hace el MIR de la dictadura militar, como la definición estratégica que se adopta (Estrategia de Guerra Popular Prolongada, EGPP). Luego el autor realiza una larga disquisición respecto de la legitimidad de la violencia política popular en contexto de dictadura para, posteriormente pasar a realizar un meticuloso análisis del movimiento de resistencia popular desarrollado por el MIR a partir de 1978.

De especial importancia para los estudios de violencia política en el período resultan los capítulos 4 y 5 (pp. 91-159), en los cuales se analizan los dos componentes fundamentales de la política militar del MIR: la Estructura de Fuerza Central (EFC) y las Milicias de la Resistencia Popular (MRP). Ambos son entendidos como elementos centrales del proceso de acumulación de fuerza que debía desarrollar el campo popular, al objeto de derrocar a la dictadura. Mientras la EFC

\footnotetext{
${ }^{30}$ Este es el caso de figuras emblemáticas del mirismo de la década de 1980, como Mauricio Maigret Becerra, muerto en 1984; Paulina Aguirre Tobar, también asesinada en 1984; los hermanos Eduardo y Rafael Vergara Toledo, asesinados por carabineros en 1985; y Aracely Romo Álvarez y Pablo Vergara Toledo, muertos en 1988.

${ }^{31}$ Pinto Vallejos, Julio, “ ¿Y la historia les dio la razón? El MIR en dictadura, 1973-1981”, en Valdivia, Verónica et al, Su revolución contra nuestra revolución. Izquierdas y derechas en el Chile de Pinochet (1973-1981), Vol. 1, Santiago de Chile: LOM Ediciones, 2006, pp. 153-205.

32 Silva Hidalgo Robinson, Resistentes y clandestinos. La violencia politica del MIR en la dictadura profunda, 1978-1982, Concepción: Escaparate, 2011. El mismo tema ha sido tratado, de manera más general, por Pérez, Cristián, "Historia del MIR. Si quieren guerra, guerra tendrán”, Estudios Públicos, 91, Santiago de Chile, 2003, pp. 5-44. Un trabajo escasamente conocido en Chile es el de Francisco García Naranjo. En este estudio el autor analiza en profundidad la historia del MIR, dedicándole un capítulo completo al despliegue de su accionar armado en contexto de dictadura. Ver, García Naranjo, Francisco, Historias derrotadas. Oposición y obstinación de la guerrilla chilena (1965-1988), Morelia: Universidad Michoacana de San Nicolás de Hidalgo, 1996, pp. 219-248.
} 
debía golpear objetivos estratégicos del aparato de seguridad de la dictadura, para demostrar la vulnerabilidad del enemigo; las MRP debían acompañar el proceso de movilización del campo popular para contribuir a la extensión de la lucha rupturista. Las diferentes manifestaciones que adoptan estos dos componentes: asaltos a instituciones financieras, ajusticiamientos de agentes de la dictadura, distribución de alimentos en poblaciones, sabotajes al tendido eléctrico, acciones milicianas urbanas, etc., dan cuenta del alcance y profundidad que alcanzó el despliegue del MIR en esta fase.

Una espiral de violencia política ascendente que, sin duda alguna, contribuyó ampliamente al posterior desarrollo de las movilizaciones populares antidictatoriales (1983-1987). Efectivamente, la movilización social del ciclo 1983-1987 no se explica sin el desarrollo previo a nivel local de formas de organización política y miliciana que estimularon y condujeron las protestas sociales. No obstante el MIR, como señala Robinson Silva, pagó un alto precio al ponerse a la cabeza de la reorganización de la lucha antidictatorial. La represión política, liderada a partir de 1977 por la Central Nacional de Informaciones (CNI), se centró en el MIR, Cientos de sus militantes fueron asesinados o murieron en enfrentamientos armados, mientras que varias centenas más fueron encarceladas y purgaron muchos años de prisión. ${ }^{33}$ El MIR, a partir de 1984 aparece como una fuerza política con un alto grado de legitimidad social y política, pero duramente golpeada en su organización interna; en especial entre sus cuadros político-militares.

Por su parte en el estudio de José Antonio Palma se abordan temas y problemáticas similares, como el contexto histórico dictatorial y las definiciones de naturaleza teórica y política que llevaron al MIR a adoptar la EGPP. ${ }^{34}$ Pero en este trabajo se realiza un análisis aún más pormenorizado y extendido de las acciones de violencia política desplegadas por el MIR, llegando a cubrir hasta el año 1988.

El autor se detiene, especialmente, a analizar las MRP, destacando no sólo el importante accionar que desplegó en el ciclo 1979-1988, sino que enfatizando la incidencia que ellas tuvieron en la configuración del movimiento popular antidictatorial (especialmente a nivel territorial), y su influencia en el desarrollo y radicalización de las protestas populares contra la dictadura. ${ }^{35}$ Este acápite del trabajo de José Antonio Palma viene a refutar, a nuestro juicio, tanto las tesis de la anomia, que identifican las protestas como un puro "reventón espontáneo de la juventud popular" (Tironi y Weinstein, previamente citados), así como las tesis "movimientistas" que pretenden establecer una autonomía absoluta de lo social respecto de lo político. En este último enfoque, las protestas populares son expresión de la reacción espontánea de los sectores populares, ancladas en un atavismo comunitario que episódicamente las impele a manifestarse contra el poder (Salazar y Garcés y De la Maza, también citados previamente).

\footnotetext{
${ }^{33}$ Los efectos de la represión sobre el MIR se pueden estudiar en Vera Quiroz, Andrés, Tortura, clandestinidad y dictadura. Una mirada desde la militancia mirista, 1982-1984, Concepción: Escaparate, 2011 y Amoros, Mario, La memoria rebelde, Concepción: Ediciones Escaparate, 2008. Un enfoque similar, pero que abarca el conjunto del ciclo dictatorial, lo desarrolla Verónica Valdivia. Ver, Valdivia Ortiz de Zarate, Veronica, "Terrorism and political violence during the Pinochet years: Chile, 1973-1989”, Radical History Review, 85, New York, 2003, pp. 182-190.

${ }^{34}$ Palma Ramos, José Antonio, El MIR y su opción por la guerra popular. Estrategia político-militar y experiencia militante, 1982-1990, Concepción: Escaparate, 2012.

${ }^{35}$ En el estudio de Julio Pinto y Sebastián Leiva, se analiza el mismo ciclo histórico, pero se pone mayor énfasis al estudio de las organizaciones de masas formadas por el MIR. Se trata de las denominadas organizaciones democrático independientes (ODIs), que jugaron un rol fundamental en la reanimación del movimiento popular. Entre ellas destacaron el Comité de Defensa de los Derechos del Pueblo (CODEPU), la Unión Nacional de Estudiantes Democráticos (UNED) y la Coordinadora de Organizaciones Populares (COAPO). En el frente de trabajadores se crearon los Comités de Trabajadores (CCTT), pero su desarrollo y extensión fue bastante menor. Ver, al respecto, Pinto, Julio y Leiva, Sebastián, "Punto de quiebre. El MIR en los ochenta", en Valdivia, Verónica et al, Su revolución contra nuestra revolución. La pugna marxista-gremialista en los ochenta, Vol. 2, Santiago de Chile: LOM Ediciones, 2006, pp. 83-138
} 
El trabajo de José Antonio Palma concluye con un análisis riguroso de la crisis política que afectó a la dirección del MIR, a partir de 1984, y que concluyó con su fractura en febrero de 1987. Para este autor, la incidencia que tuvo en la crisis la discusión del fracaso estratégico-táctico del ciclo 1978-1982 resultó determinante. Efectivamente, los balances realizados al efecto por los plenos del Comité Central realizados en La Habana, dieron cuenta de la profundidad de la derrota de la organización. Este ejercicio evaluativo, con matices compartidos, dio origen a propuestas de salida política que provocaron la escisión. No obstante, la profunda debilidad de todas las fracciones convirtió en inviables todas las opciones. El MIR se precipitó, de esta manera a su colapso definitivo ${ }^{36}$.

No obstante el importante avance que ofrecen los estudios sobre el MIR, la fase 1973-1994 continúa presentando importantes déficits. A nuestro juicio es necesario analizar en profundidad las características operativas que asumió la Operación Retorno (uno de los hitos más emblemáticos y controversiales en la historia del MIR). También es necesario cuantificar y evaluar de manera más precisa su accionar operativo y la incidencia política del mismo. Otro aspecto que no cuenta con estudios historiográficos rigurosos es el proyecto de asentamiento guerrillero en las zonas de Neltume y Nahuelbuta. Este episodio, que ha dado origen a duras polémicas al interior del mirismo y en el ámbito de la izquierda en Chile, requiere un trabajo monográfico que aún se encuentra pendiente. ${ }^{37}$ De la misma manera, se conoce poco respecto de la participación de las MRP en las protestas masivas del ciclo 1983-1987. Y prácticamente no se conoce nada sobre los rasgos distintivos de la crisis interna del período 1984-1988 o sobre la fragmentación y colapso de la organización durante la transición temprana (1990-1994).

\section{Los estudios sobre la Política de Rebelión Popular de Masas del Partido Comunista}

La relación entre el PC y su brazo armado, el Frente Patriótico Manuel Rodríguez (FPMR), con la definición y puesta en práctica de la violencia política se encuentra mediada por la denominada "política de rebelión popular de masas" (PRPM), temática que también ha concitado el interés de los investigadores. ${ }^{38}$ Uno de los aportes más interesantes es el de Rolando Álvarez, que da cuenta tanto de los alcances políticos de la renovación por la izquierda de los comunistas chilenos, a partir de 1980, como de las tensiones que el giro armado provocó al interior del PC. ${ }^{39}$ Efectivamente, en su trabajo, Rolando Álvarez enfatiza que la PRPM fue el resultado de varios factores. Por una parte, de la derrota político-militar de la experiencia de la UP, que puso de manifiesto las falencias de la política militar de la izquierda chilena. Por otro lado, de la instalación en Cuba y en la República Democrática Alemana (RDA), de un importante contingente de comunistas chilenos que

\footnotetext{
${ }^{36}$ A su vez, en nuestros trabajos hemos enfatizado en el rol que le cabe a la violencia en el diseño estratégico de esta organización y en la crisis y deriva cultural de la misma a partir de 1986. Ver, Goicovic Donoso, Igor, "El Movimiento de Izquierda Revolucionaria (MIR) y la irrupción de la lucha armada en Chile, 1965-1990", en Pozzi, Pablo y Pérez, Claudio (Editores), Historia oral e historia politica. Izquierda y lucha armada en América Latina, 1960-1990, Santiago de Chile: LOM Ediciones - UAHC, 2012, pp. 159-189; Goicovic Donoso, Igor, "Transición y violencia política en Chile (1988-1994)", Ayer. Revista de Historia Contemporánea, 79: 3, Madrid, 2010, pp. 59-86; y Goicovic Donoso, Igor, "Violencia y poder en la estrategia del Movimiento de Izquierda Revolucionaria, 1967-1986", Cuadernos Sociológicos, 3, Santiago de Chile, 2004, pp. 171-183

37 No obstante existe un muy buen relato testimonial. Ver, Comité Memoria Neltume, Guerrilla en Neltume. Una historia de lucha y resistencia en el sur chileno, Santiago de Chile, LOM, 2003.

${ }^{38}$ Una síntesis muy completa con las principales referencias bibliográficas sobre la Historia del Partido Comunista de Chile en Álvarez Vallejos, Rolando, "Historias, historiografía y memorias del comunismo chileno en la primera década del siglo XXI. Un ensayo bibliográfico”. Este artículo es el prólogo al libro de Viviana Bravo, ¡Con la razón y la fuerza venceremos! La rebelión popular y la subjetividad comunista en los '80, Santiago de Chile: Ariadna Ediciones, 2010, pp. 11-30.

39 Álvarez Vallejos, Rolando, Arriba los pobres del mundo. Cultura e identidad politica del Partido Comunista de Chile entre democracia y dictadura, 1965-1990, Santiago de Chile: LOM Ediciones, 2011. Previamente Rolando Álvarez, había analizado el complejo proceso de clandestinidad del PC entre 1973 y 1980. Ver, Álvarez Vallejos, Rolando, Desde las sombras. Una bistoria de la clandestinidad comunista (1973-1980), Santiago de Chile: LOM Ediciones, 2003.
} 
experimentó un proceso de radicalización teórica y política. También influyó la formación en la RDA de un equipo de intelectuales (Grupo de Leipzig), que comenzó a desarrollar una reflexión teórica e histórica sobre las FFAA, la sociedad y la izquierda chilena. Y, por último, el giro hacia la izquierda del PC, derivado del fracaso de la política del Frente Antifascista que se había impulsado entre 1974 y 1979.

De la misma manera, el trabajo de Luis Rojas Núñez, levantado con fuentes poco conocidas (algunas de ellas obtenidas en Cuba), permite conocer en detalle la relación política entre el PC y el FPMR a través de la PRPM. ${ }^{40}$ En uno de los apartados de su libro (pp. 11-89, "Los años urgentes"), se describe con precisión los hechos de violencia política protagonizados por estas organizaciones en el contexto de las protestas populares de la década de 1980.

La estructura territorial del FPMR se extendía desde la región de Atacama por el norte, hasta la región de la Araucanía por el sur. Su unidad de base eran los grupos operativos, los cuales se organizaban en columnas y éstas, a su vez, se encontraban adscritas a destacamentos. Existían un total de cuatro destacamentos, dos en Santiago, otro en el norte y el último en el sur del país. Existía, también, un destacamento o fuerza especial que dependía directamente del comandante del FPMR, Raúl Pellegrin Friedman, más conocido como "José Miguel", muerto en un enfrentamiento armado en la localidad de Los Queñes (región del Maule), en octubre de 1988. Esta unidad destacaba por la originalidad y espectacularidad de las acciones armadas desplegadas: Tomas de radioemisoras para emitir proclamas antidictatoriales; secuestros a representantes del régimen militar; distribución de alimentos en poblaciones populares; y ataques con explosivos y armas automáticas a unidades policiales. ${ }^{41}$

No obstante el alto grado de especialización del FPMR y el importante nivel de calificación operativa de sus cuadros (muchos de ellos formados en las Fuerzas Armadas Revolucionarias, FAR, de Cuba), el principal soporte de la actividad armada del FPMR lo constituyó la ingente base social de apoyo que le brindaba el PC. Efectivamente el PC chileno, a pesar de la represión política, seguía siendo en la década de 1980 un partido disciplinado y sólidamente arraigado en el movimiento popular chileno. Ello le permitió al FPMR disponer, a lo largo de todo Chile, de una amplia red de colaboradores, ayudistas, simpatizantes y milicianos que contribuían, cada uno de acuerdo con sus capacidades y disposición, al desarrollo de la PRPM. De la misma manera esta red facilitaba, casas de almacenamiento, recursos materiales y financieros y coberturas de seguridad. Sobre la base de estos antecedentes, el libro de Jorge Rojas demuestra que la PRPM no fue la concatenación de una serie de maniobras conspirativas dotadas de un alto grado de especialidad técnica, sino que el despliegue de un trabajo partidario que involucró al conjunto de la organización y a la mayoría de los frentes de masas que le eran afines.

Por su parte, el trabajo de Viviana Bravo, también coloca el acento en la definición de la estrategia y en el desarrollo de la acción armada en el contexto de la década de 1980, pero destacando las convicciones militantes que rodearon el nuevo quehacer del PC. ${ }^{42}$ En este punto Viviana Bravo apela a la noción de indignación, como una condición fundamental de repudió a la dictadura y de disposición a la acción antidictatorial. Esta indignación, que opera como el factor fundamental en las motivaciones que llevan a los militantes a tomar las armas contra el régimen, derivaba de dos construcciones subjetivas fundamentales: La internalización de la derrota de la Unidad Popular (1973) y las implicancias de esa derrota en el entorno familiar y social en el cual

\footnotetext{
${ }^{40}$ Rojas Núñez, Luis, De la rebelión popular a la sublevación imaginada. Antecedentes de la historia política y militar del Partido Comunista de Chile y del FPMR, 1973-1990, Santiago de Chile: Ariadna Ediciones, 2011.

${ }^{41}$ Una de las acciones más espectaculares del FPMR en este período fue la denominada "Operación Príncipe", que consistió en el secuestro, por 92 días, del coronel de Ejército, Carlos Carreño Barrera (entre el 1 de septiembre y el 3 de diciembre de 1987). Carreño, director en esa época de la Fábrica y Maestranza del Ejército (FAMAE), fue secuestrado en Santiago y posteriormente liberado en la ciudad brasileña de Sao Paulo.

${ }^{42}$ Bravo, Viviana, ;Con la razón y la fuerza venceremos! La rebelión popular y la subjetividad comunista en los '80, Santiago de Chile: Ariadna Ediciones, 2010.
} 
se desenvolvía el sujeto; y la percepción de la dictadura como un régimen represivo al servicio de los patrones. Estos aspectos, centrales en la configuración de las subjetividades que acompañan la opción por la militancia armada, tienden a coincidir, en el caso de los militantes del FPMR, con la existencia de una tradición familiar comunista, que alentaba o protegía la participación en la organización.

Por otro lado, los cambios en la línea política del PC y las consideraciones que rodearon el debate y ejecución de la PRPM han sido testimoniados ampliamente en la compilación de Manuel Loyola y Jorge Rojas. ${ }^{43}$ Cesar Quiroz (pp. 247-258), por ejemplo, sostiene (apegándose al discurso oficial del PC), que la PRPM sólo fue una adecuación táctica de la política tradicional del PC. ${ }^{44}$ Esta adecuación táctica, derivada del escenario impuesto por la dictadura, habría consistido en: Una profundización de los aspectos militares; la introducción de una disposición nueva orientada a disputarle a la burguesía el monopolio de la fuerza; y el diseño de una nueva concepción sobre el recurso a la violencia en el plano político. En una línea de análisis similar Oscar Azocar (pp. 261275), complementa que la PRPM conllevaba una salida popular a la crisis política de la dictadura. Efectivamente, los bloques políticos opositores constituidos hacia comienzos de la década de 1980 (la Alianza Democrática y el Movimiento Democrático Popular), habían definido con claridad sus itinerarios políticos. Para la Alianza Democrática (AD), liderada por la Democracia Cristiana e integrada por las diferentes expresiones del socialismo renovado, resultaba prioritario avanzar de manera pacífica hacia una transición a la democracia, lo cual hacia inevitable pactar o negociar con la dictadura o sus representantes. Mientras que para el Movimiento Democrático Popular (MDP), liderado por el PC, e integrado por el MIR y el PS dirigido por Clodomiro Almeyda, se imponía una salida rupturista a la dictadura, que garantizara la inclusión de los sectores populares y sus demandas. En este escenario, a juicio de Azocar, la PRPM generaba las condiciones políticas para la salida rupturista y popular a la dictadura.

Similar problema aborda Alfredo Riquelme, pero asentado en una perspectiva crítica, que pone en evidencia el aislamiento que supuso para el PC la adopción de la PRPM. ${ }^{45}$ En su estudio Riquelme devela este proceso como parte de la revisión e ideologización vividos por el PC, tras la derrota de 1973.

Hernán Vidal, en otro enfoque crítico, sostiene que en relación al FPMR y al accionar armado en Chile, se ha construido un tabú, concordado implícitamente entre los detentadores del poder (transición a la democracia) y quienes se le opusieron al mismo (el FPMR). ${ }^{46}$ Este tabú pretende, en un caso, invisibilizar el accionar el armado, en cuanto este cuestiona las bases del sistema político en construcción y, en el otro campo, dificulta la inserción social y política de los guerrilleros derrotados. El tabú, para Vidal, sería entonces parte de la neurosis social que afecta a la sociedad chilena y que deriva del trauma colectivo provocado por el golpe de Estado de 1973 y el posterior régimen dictatorial. En este esquema de análisis, la lucha armada fue un ejercicio político inconducente, que sólo habría contribuido a profundizar dicho trauma.

Por su parte, Carmelo Furci, en un estudio más longitudinal sobre el PC, estableció que la radicalización de los comunistas chilenos fue el resultado del fracaso de la política de "Frente Antifascista" enarbolada originalmente por el PC. ${ }^{47}$ Efectivamente, el proceso de institucionalización de la dictadura a partir de 1977 y la tendencia del centro político a buscar pactos o acuerdos con ésta, hicieron inviable la política de "Frente Antifascista", arrastrando al

\footnotetext{
${ }^{43}$ Loyola, Manuel y Rojas, Jorge (compiladores), Por un rojo amanecer. Hacia una bistoria de los comunistas chilenos, Santiago de Chile: Impresora Valus, 2000.

${ }^{44}$ La línea política del PC, desde 1956 en adelante, habría estado sostenida en: La unidad de la clase trabajadora y el pueblo, la lucha por conquistas sociales y políticas y la unidad socialista-comunista.

45 Riquelme, Alfredo, Rojo atardecer. El comunismo chileno entre dictadura y democracia, Santiago de Chile: DIBAM, 2009.

46 Vidal, Hernán, FPMR. El tabú del conflicto armado en Chile, Santiago de Chile: Mosquito Editores, 1995.

${ }^{47}$ Furci, Carmelo, El Partido Comunista de Chile y la vía al socialismo, Santiago de Chile: Ariadna Ediciones, 2008, pp. 248-252.
} 
PC a la definición de una política rupturista (uso de todas las formas de lucha), en la cual sólo se encontró con el MIR y con una fracción del PS. En este enfoque, la adopción de la PRPM fue el resultado del itinerario político instalado por la dictadura y del creciente aislamiento experimentado por el PC chileno.

Un campo especialmente interesante y novedoso es el de la internacionalización de las organizaciones armadas chilenas. Como lo demuestra un reciente estudio de Claudio Pérez, el PC no sólo definió la necesidad de formar cuadros militares para el nuevo escenario político que se habría hacia fines de la década de 1970 en Chile, sino que también los desplegó operativamente en Centroamérica (especialmente en Nicaragua) al objeto que éstos adquirieran experiencia de combate. ${ }^{48}$ De esta manera, un numeroso contingente de chilenos, especialmente comunistas y socialistas, participó activamente del proceso armado que llevó al triunfo de los sandinistas en julio de 1979 y que posteriormente supuso la defensa del proceso revolucionario frente a la agresión de los grupos contrarrevolucionarios amparados por EE.UU. La mayoría de estos combatientes poseían formación especializada en el arma de artillería y colaboraron activamente en el denominado Frente Sur, que operaba desde la frontera con Costa Rica

Como señalamos previamente, el análisis historiográfico sobre el PC cuenta, sin duda alguna, con importantes contribuciones. Al trabajo referencial de Hernán Ramírez Necochea de 1984, es imprescindible sumar las múltiples contribuciones de Rolando Álvarez y, más recientemente el trabajo de Alfredo Riquelme. ${ }^{49}$ Sin embargo, la historiografía sobre el FPMR sigue siendo escasa. Múltiples aspectos de esta organización, como el desempeño de sus militantes en las guerras civiles centroamericanas, las tensiones entre partido y aparato armado, la escisión del aparato armado en 1988, el diseño y puesta en ejecución de la Guerra Patriótica Nacional (GPN) y el colapso de la organización en la transición temprana, requieren, también, de mayores niveles de profundización.

\section{Los estudios sobre el MAPU Lautaro}

De los tres referentes políticos más importantes en el despliegue de acciones de naturaleza violenta, el Movimiento de Acción Popular Unitaria (MAPU) Lautaro es el que cuenta con menos investigaciones disciplinarias. Una primera aproximación a este tema lo realizó Cristina Moyano, quien sostuvo que la emergencia del MAPU Lautaro se inscribe en el contexto más amplio de la renovación del socialismo chileno. ${ }^{50}$ Efectivamente, conceptos como rebeldía popular y movimiento social, se encuentran a la base de las reflexiones que el MAPU comenzó a desarrollar después del golpe de Estado de 1973 y que, posteriormente (V Pleno del Comité Central, 1983), un sector de su Comisión Juvenil, utilizó para afirmar la necesidad de la lucha armada como expresión de ruptura política.

Este sujeto juvenil no tenía cabida en la izquierda tradicional y ni siquiera era suficientemente entendido o asimilado por la izquierda armada de matriz castro-guevarista. De esta manera, el MAPU Lautaro se convirtió en la síntesis entre pueblo joven y revolución. Un pueblo, que dado el contexto que se vivía, requería fundamentalmente de acción (no de teorías). La política,

\footnotetext{
48 Pérez Silva, Claudio, "De la guerra contra Somoza a la guerra contra Pinochet. La experiencia internacionalista revolucionaria en Nicaragua y la construcción de la fuerza militar propia del Partido Comunista de Chile", en Pozzi, Pablo y Pérez, Claudio (Editores), Historia oral e historia politica. Izquierda y lucha armada en América Latina, 1960-1990, Santiago de Chile: LOM Ediciones - UAHC, 2012, pp. 213-244. Ver, también, Pérez, Cristián, “Compañeros a las armas: Combatientes chilenos en Centroamérica (1979-1989”, Estudios Públicos, 129, Santiago de Chile, 2013, pp. 141 164.

${ }^{49}$ Ramírez Necochea, Hernán, Origen y formación del Partido Comunista de Chile. Ensayo de bistoria politica y social de Chile, Moscú: Progreso, 1984.

${ }^{50}$ Moyano Barahona, Cristina, El MAPU durante la dictadura. Saberes y prácticas políticas para una microbistoria de la renovación socialista en Chile, 1973-1989, Santiago de Chile: Ediciones Universidad Alberto Hurtado, 2010, pp. 391-420.
} 
entonces, dejó de ser posibilidad y pasó a convertirse en voluntarismo. Por esta vía la acción revolucionaria del Lautaro, expresada fundamentalmente como asaltos a instituciones financieras, ocupaciones territoriales y repartición de alimentos (Copamientos Territoriales Armados, CTA) y ejecución de funcionarios y de carabineros, se convirtió en la concreción de los sueños y las ganas del mundo popular, que aspiraba a tener acceso a los bienes que el capital les negaba y a disfrutar de la libertad que el Estado les restringía.

La monografía más acuciosa sobre el tema es la de Ivette Lozoya. ${ }^{51}$ En su trabajo la autora sostiene que el MAPU Lautaro optó por construirse en el seno del mundo popular, y en especial entre los jóvenes urbano-populares. El sujeto más golpeado por la represión y la exclusión social y económica. De esta manera el MAPU Lautaro alcanzó un importante nivel de crecimiento hacia fines de la década de 1980 y comienzos de la década de 1990; fase que coincide con su mayor capacidad de intervención operativa. No obstante, el accionar represivo, en especial en el ciclo 1991-1994, redujo la capacidad operativa de su estructura especializada (las Fuerzas Rebeldes y Populares Lautaro, FRPL) y afectó el despliegue de su estructura de masas (Movimiento Juvenil Lautaro, MJL). A partir de 1993 las estructuras orgánicas del MAPU Lautaro se encuentran muy debilitadas y la moral militante resquebrajada. De esta manera, la captura de su máximo dirigente, Guillermo Ossandon Cañas, en junio de 1994, marcó el inicio del colapso para la organización.

Otros trabajos, a nivel de tesis de grado, profundizan en las subjetivaciones de la lucha armada y en las experiencias y resignificaciones que los militantes lautaristas han realizado de esas etapas de sus vidas. ${ }^{52}$

Al igual que en los casos anteriores los déficits para el caso del MAPU Lautaro son extraordinariamente evidentes. Aún desconocemos todo sobre la génesis del movimiento. Tampoco existen estudios acabados sobre su estructura orgánica y sobre su diseño estratégico. De la misma manera las incógnitas sobre su accionar político, su relación con el PC y el resto de la izquierda y la ejecución de su política militar sólo cuentan con estudios exploratorios.

\section{Conclusión}

De acuerdo a lo establecido por Pedro Rosas, este largo ciclo de violencia insurgente (1978-1994) enfrentó una dura réplica por parte del Estado. ${ }^{53}$ Hacia fines de la década de 1970 la dictadura militar desplegaba una estrategia represiva de tipo selectivo, que apuntaba a impedir la rearticulación de la izquierda revolucionaria. Para ello las ejecuciones extrajudiciales, la tortura y el encierro prolongado se convirtieron en los principales mecanismos punitivos. Estos métodos no experimentaron modificaciones importantes durante la transición temprana (1990-1994). Pero si fue modificada la estructura de seguridad (desapareció, por ejemplo, la Central Nacional de Informaciones, CNI), adquiriendo Carabineros y la Policía de Investigaciones mayores atribuciones en la lucha contra el terrorismo. Mientras que en el plano político, la defensa de la institucionalidad democrática, le otorgó mayor legitimidad a la represión.

\footnotetext{
${ }^{51}$ Lozoya López, Ivette, "Chile: Violencia política y transición a la democracia. El MAPU Lautaro y la derrota de la vía revolucionaria en los 90", en Pozzi, Pablo y Pérez, Claudio (Editores), Historia oral e historia política. Irquierda y lucha armada en América Latina, 1960-1990, Santiago de Chile: LOM Ediciones - UAHC, 2012, pp. 191-212

${ }^{52} \mathrm{Ver}$, al respecto, Rosas Aravena, Pedro, Por la senda de Lautaro. Latinoamérica vencerá. Discurso, acción política, concepción y dimensión internacional del MAPU Lautaro, Tesis de doctorado, Doctorado en Estudios Americanos, Santiago de Chile: Universidad de Santiago de Chile, 2012; Briceño Ramírez, Laura, La revolución aquí y abora. Juventud popular y militancia revolucionaria. Una mirada desde el MAPU Lautaro (1983-1994), Tesis para optar al grado de licenciado en Historia, Santiago de Chile: Universidad de Santiago de Chile, 2011; y Acevedo Arriaza, Nicolás, Fuera Pinochet. Chile popular. El MAPU Lautaro en las protestas populares (1978-1985) Tesis para optar al grado de Licenciado en Historia, Santiago de Chile: Universidad ARCIS, 2006. Un excelente trabajo de difusión respecto de la historia del MAPUA Lautaro en, Acevedo, Nicolás, MAPU - Lautaro, Concepción: Escaparate, 2014.

${ }^{53}$ Rosas Aravena, Pedro, Rebeldía, subversión y prisión política. Crimen y castigo en la transición chilena, 1990-2004, Santiago de Chile: LOM Ediciones, 2004.
} 
El ciclo de la violencia insurgente se cerró hacia 1994 con la derrota completa y extensiva de las organizaciones armadas. No obstante, sus militantes de base se difuminaron al interior de la sociedad y, al momento del repunte del movimiento popular (2006), continuaban teniendo participación política y ejerciendo un fuerte liderazgo entre las organizaciones juveniles secundarias, universitarias y populares. No es extraño, entonces, que sus símbolos, sus líderes y héroes, sus consignas e incluso algunos niveles de su intervención miliciana, sean recuperados y reinstalados por diferentes grupos revolucionarios contemporáneos.

La historia de la insurgencia armada en Chile, es parte de la historia política reciente de ese país. En consecuencia, profundizar en su estudio y análisis nos permitirá conocer un poco más de las tensiones y conflictos de la sociedad en su conjunto, e incluso de sus proyecciones hacia el tiempo presente. 